\title{
Indocyanine Green Fluorescence in Surgical Practice - A Narrative Review of Clinical Applications
}

\author{
Catalin ALIUS ${ }^{1}$, Eugen-Sebastian GRADINARU ${ }^{1}$, Adriana Elena NICA ${ }^{2}$ \\ $14^{\text {th }}$ Department of Surgery. Bucharest University Emergency Hospital, Romania \\ ${ }^{2} 2^{\text {nd }}$ Intensive Care Unit, Bucharest University Emergency Hospital, Romania
}

\section{ABSTRACT}

The introduction in surgical practice of NIR fluorescent systems with their augmented reality enhanced the visual abilities of the surgeons who imagined over just a decade a myriad of uses for this emergent technology. We reviewed the clinical applications of Indocyanine Green from intraoperative cholangiography and SLN identification to bowel perfusion assessment, endocrine surgery and evaluation of Crohn's disease and Barrett's esophagus. The immense value of this new method of functional and anatomical assessment is unequivocal, but there are pitfalls and drawbacks of the commercially available systems. By presenting all these we hope to disseminate the importance and the potential of the technology and to promote the interest in usage and research in the field.
\end{abstract}

Keywords: fluorescence, Indocyanine green, surgery

\section{INTRODUCTION}

Recently emerging technologies such Near Infrared Fluorescence Imaging Systems are becoming a major contributor to increased performance in the operating theatre and help rethinking safety paradigms (1). Despite an accelerated advance in the medical imaging filed, data obtained with CTs, MRIs, PET scans and ultrasounds have a very limited role during the operations, hence the clinical need to enhancing the visual abilities of the surgeon beyond what can be seen with the naked eye in order allow for better intraoperative decision making and decreased rates of complications (2). NIR Fluorescence Imaging systems have been proposed to meet this requirement and gained momentum in the past decade since the development of the commercially available systems because they are affordable and safe to use. As a consequence, a myriad of uses have been proposed. The technology uses a fluorescent dye emitting NIR light (which is capable of tissue penetration up to $1 \mathrm{~cm}$ ) (4) and overlaps conventional imaging with digitalized data from the NIR spectrum in order to achieve augmented reality images during surgery (5). The technology has been described over 70 years ago but its importance was rediscovered only in the last decade when experimental surgery used it to acquire functional and anatomical data $(5,6)$.

ICG enhanced surgery is used for delineation of anatomical structures especially in the porta hepatis region and in vascular surgery for identification of small vessels. In plastic surgery for assesment of pedicles for flap selection and wound characterization, in breast surgery it evaluates skin flaps and facilitates sentinel lymph nodes identification. Colorectal surgery uses ICG to demonstrate viability of the bowel, 
to evaluate the blood supply in anastomoses, to identify locoregional lymps nodes, for tattooing of the peritumoral tissues, for extralumenal staining of the mesorectal fat in low rectal interventions etc. In upper GI surgery this technology offers additional information on the biliary anastomoses, pancreatic stumps, functional information about the liver, details on its segmentation, intraoperative cholangyography and tumour localization. An interesting use is in delineating the resection margins of the cystic tumours developed in well vascularized tissues, especially the liver. Apart from these, ICG enhanced imaging offer unprecedented details when employed in lymphadenectomy, and endocrine surgery for identification of the parathyroid glands and the adrenals. New ICG labeled molecules are being tested to expand the spectrum of indications to identification of carcinomatosis, small tumoral deposits, metaplasia and for assessment of inflammatory bowel diseases. We first discuss the properties of the ICG and the NIR system followed by a more detailed presentation of the most important clinical applications summarizing at the end the pitfalls and challenges posed by this revolutionary technology which laid dormant for three quarters of a century only to emerge rapidly over the past 8-10 years after it has been linked to the progress in the engineering domains (7-35).

\section{ICG PROPERTIES AND OPTICAL SYSTEMS}

\section{ICG}

Indocyanine Green is a disulfonated heptamethine indocyanine with a molecular mass of 776 kDa developed in 1955 and FDA approved since 1959 (4). This molecule is injected into the blood stream and binds rapidly to plasma proteins having thus minimal interstitial leakage. Its half life in the blood stream is between 3 and 5 minutes because it is extracted by the liver which starts to excrete it in the bile after 10-15 minutes (7). Up to date there are no known metabolites of ICG identified. Some interesting research is conducted in the direction of measuring the variation of the fluorophore's life time when bound to different molecules (8). It generates fluorescence at around 830-845 nm when exposed to NIR light with a peak absorption at $805 \mathrm{~nm}(9,10)$. The fluorescence measured in vivo manifest in 3-5 seconds from the injection moment for the vascular structures (and lasts for a short period of time) and at 1015 minutes in the bile (and lasts for hours). Since the introduction of this technology various studies determined the fluorescence intensity in different moments of the surgery in relation to different doses of the fluorophor molecule. Few studies explored different doses starting from 0.02 to $0.25 \mathrm{mg} / \mathrm{kg}$ and measured intensities of the liver, fat and bile duct establishing that the best intensity is achieved with $0.25 \mathrm{mg} /$ $\mathrm{kg}$ (11). Another study measured fluorescent intensities at different intervals between 1 to 24 hours and concluded that administration of ICG at 3 hours before the surgery offers the best imaging experience (12).

\section{Near Infrared Fluorescence Imaging Systems}

A NIR fluorescence system has to provide augmented reality images by overlapping high definition images in the visual field with digitalized fluorescence data. NIR spectrum images are invisible "per se", but with adequate software the conventional images can be augmented through discoloration of certain areas, glowing effect over some structures or gradients.

The first NIR imaging systems became commercially available around 15 years ago and ever since they are being constantly updated. Regardless of its make any NIR system need to be able to: 1 - overlay in real time white light reflectance and fluorescence images, 2 - allow operation of fluorescence mode with ambient room lightning, 3 - have high sensitivity to the fluorophore of interest, 4 - have the ability to quantify flourophores in situ and, if possible, 5 to quantify multiple flourophors simultaneously. The key components must include: 1 - a light source for the excitation light, 2 - light collection optics and filters, 3 - camera, 4 - instrument control acquisition and display software and 5 - computing, input and display hardware (13).

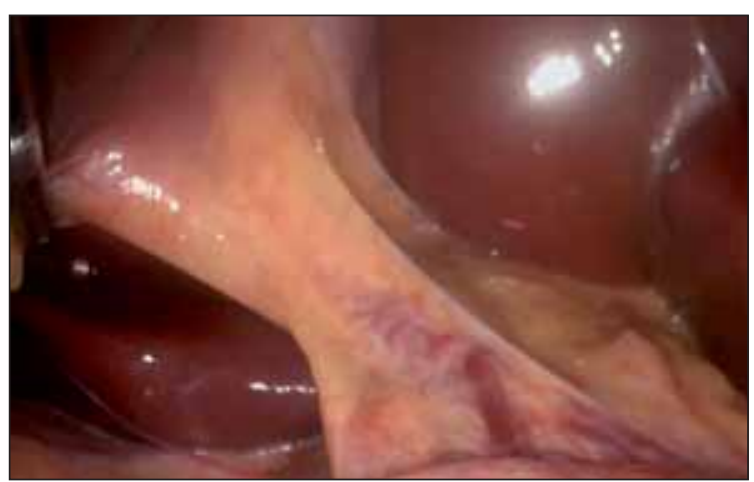

FIGURE 1. Conventional imaging of the cystic duct and the CBD under fatty tissue 


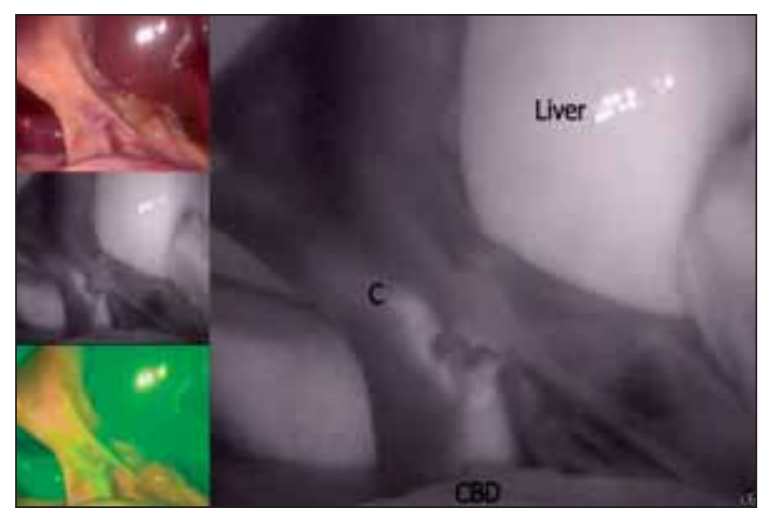

FIGURE 2. Main image showing the biliary tree in digitalized NIR imaging; Image in the upper left corner shows conventional imaging; Image in the lower left corner shows the biliary tree in augmented reality

\section{CLINICAL USES OF THE METHOD}

The potential applications of this technology could reduce the morbidity and mortality rates by lowering complications, raising curability rates and facilitating more accurate surgeries.

\section{Colorectal Surgery}

ICG is used in colorectal surgery to assess the bowel before formation of anastomoses. Because the naked eye cannot provide accurate functional information between 6 and $8 \%$ of the colorectal anastomoses will leak generating morbidity and mortality $(14,15,16)$. One author reported in a number of 38 consecutive colorectal anastomoses assessed with ICG no leaks. Furthermore he had to refashion two of the anastomoses because of poor ICG perfusion despite normal macroscopic appeareance of the bowel (4). A large reduction in the number of fistulous complications in colorectal surgery has been reported systematically by authors who employed NIR assessment of the bowel (7). Apart from this application, evaluation of an ischemic loop of intestine trapped in a strangulated hernia could precipitate a change in the surgical strategy. Some authors reported resections indicated by NIR assessment in segments of bowel which looked viable (17).

Operating times could be lowered by using preoperative endoscopic tattooing of the tumoral areas. The peritumoral injection is not only a good tattooing agent but if diluted in 30\% albumin solution it will get absorbed slowly into the lymphatic system and will provide a navigation pathway for lymphadenectomy. This application will increase survival by promoting a better adherence to the oncological principles. The principle is more eloquent in gastric surgery where there is a large density of lymph nodes stations positioned in very close proximity to each other (18).

\section{Oncological care}

Extravasation injuries are conditions resulting secondary to injection of different substances paravenously. It is quite common for the oncological catheters do dislodge and to generate extravasation injuries. Avoidance of increased comorbidity related to irritant and vesicant chemotherapy agents has been reported by using ICG angiography in selected cases (19).

\section{Plastic Surgery and Oncoplastic Breast Surgery}

ICG video angiography is a reliable method in plastic surgery for assessing the tissue perfusion in peripheral organs in order to decide on the viability of the grafts, need and extent of tissue debridement and surgical strategies. It is also of importance in any type o transplantation, selection of flap pedicles and identification of perforator vessels $(20,21,22)$. Roles for the ICG in the postoperative evaluation of grafts, flaps (including latissimus dorsi reconstructions) have been described (23-26).

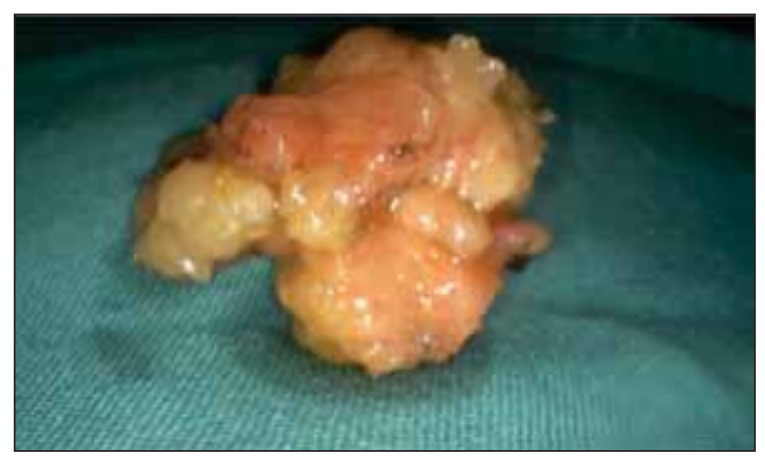

FIGURE 3. Conventional image showing a SLN

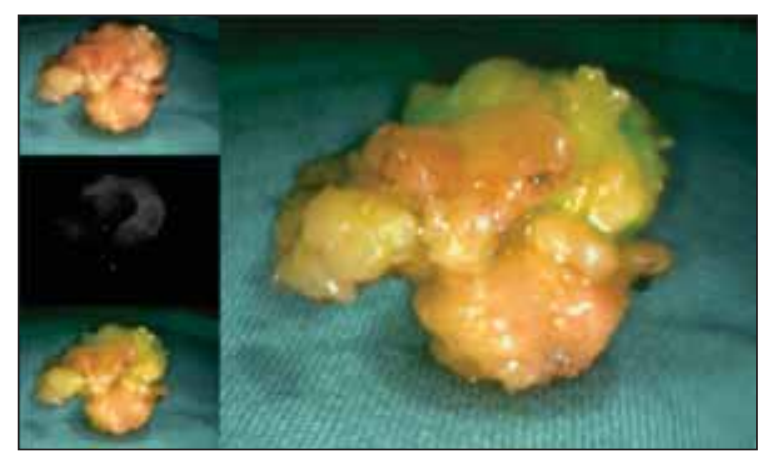

FIGURE 4. Main image showing a SLN in augmented reality; Image in the upper left corner shows conventional imaging; Middle image on left shows a SLN in digitalized NIR 
The progress from radical mastectomies to simple mastectomies and sentinel lymph node biopsy (SLN) followed or not by axillary clearance was promoted by evidence based clinical research and the need to reduce postoperative morbidity in breast cancer patients. Accurate identification of SLN could prompt a complete axillary clearance during the same operation (if immediate cyosectioning is available) or at a later stage. Several studies have demonstrated that ICG can be used to both node mapping and identification of SLN reducing the operating times and having superior results to Methylen blue (27).

\section{Upper GI surgery}

Perhaps one of the most commonly encountered uses of ICG is the identification of biliary tree anatomy. This is facilitated by its excretion (99\%) and persistence in the bile ducts and liver for hours. Several studies showed reduced operating times and safer surgeries with the identification of the vessels and ducts under the fatty tissues in the triangle of Calot. This application has allowed the challenge of old safety paradigms according to which surgery in certain acute biliary conditions were prohibited $(28,29)$. Liver mapping, intraoperative cholangiography, tumour visualization and partial liver graft evaluation are some indication in liver surgery $(30,31,32)$. A French author identifies during a Whipple procedure an ischemic segment of the pancreatic remnant before construction of a pancreaticojejunal anastomosis, prompting refashioning of its margins. The case is reported with an emphasis on the importance of NIR fluorescence in preventing a catastrophic complication, albeit no macroscopic changes were present. Cystic liver tumours are very challenging to operate because the delineation between the wall of the cyst and the liver is not easily seen. To avoid unnecessary traction and displacement of vessels or rupture of the cyst wall ICG has been used to create contrast between the liver tissue and the paucivascular cystic wall (33).

\section{Endocrine Surgery and Miscellaneous indications}

According to Kahramangil in his most recent review paper from 2017 about the use of near infrared fluorescence imaging in endocrine surgery finding the exact dissection plan is crucial for endocrine operations. ICG is useful in identifying the glowing adrenal glands under the fatty tissue that surround them and further more finding its vascular supply. In parathyroid surgery the rich vascular supply of the glands pinpointed their location after ICG injection. The method is even more efficient in thyroidectomized patients because it eliminates the background fluorescence noise (34).

A myriad of possible indications of ICG have been proposed in the past decade thanks to the improvement of CCD cameras, light sources and optical systems which increased the sensitivity of the technique. From staging and metastasis demonstration to assessment of Crohn disease and Barrett's oesophagus, ICG enhanced NIR imaging opens new perspectives in the surgical field.

\section{CONCLUSIONS}

The human eye can only offer rough macroscopic information on tissues, being a poor functional assessor and with limitation to the surfaces of the areas examined. The introduction of the NIR fluorescent systems with their augmented reality enhanced the visual abilities of the surgeons who became more accurate and able to treat old conditions in a new way. We have presented a multitude of indications for the ICG including: SLN identification, colorectal vascular assessment, flap and graft evaluations, staging of tumors, localization of endocrine glands, metastatic deposits, tattooing, perforators, wound assessment and extravasation injuries. NIR fluorescence it not an infallible technology and like any emergent method it needs to be perfected in order to meet the growing demands of its utilizers. The intensity of the fluorescence achieved is very low in certain conditions (background noise) and the maximum penetration does not exceed $1 \mathrm{~cm}$, meaning that in very obese patients or in tissues buried under fatty surroundings the technology would not be as reliable. Injecting the dye is a hazardous event although allergic reactions are much rarer than with the CT or MRI dyes. Patients with thyroid diseases and those with iodine allergy cannot benefit from the ICG based technology which is not even very widely spread. We recognize the potential of this emerging technology but we also need to contemplate on the pitfalls and challenges associated with it. The large number of clinical applications and the interest showed so far will undoubtedly attract more and more researchers and clinicians into using and developing the NIR fluorescence medical systems. 


\section{REFERENCES}

1. Ankersmith M., Van Dam D., Van Rijswijk A.S. et al. Fluorescent imaging with Indocyanine Green During Laparoscopic Cholecystectomy in Patients at Increased Risk of Bile Duct Injury. Surgical Innovation 2016; 23(4):360-36

2. Labadie R.F., Davis B.M., Fitzpatrick J.M. 2005. Image-guided surgery: What is the accuracy? Curr. Opin. Otolaryngol. Head Neck Surg. 13:27-31

3. Gioux S., Soo Choi H., Frangiony J.V. Image Guided Surgery using Invisible Near Infrared Light: Fundamentals of Clinical Translation. Mol. Imaging. 2010; 9(5):237-255

4. Boni L., David G., Mangano A. et al. Clinical applications of indocyanine green (ICG) enhanced fluorescence in laparoscopic surgery. Surg Endosc 2015; 29(7):2046-2055

5. Novotny H., Alvis D. 1960. A method of photographing fluorescence in circulating blood of the human eye. Tech. Doc. Rep. SAM-TDR. USAF Sch. Aerosp. Med. 60:1-4

6. Moore G., Hunter S., Hubbard T. 1949. Clinical and experimental studies of fluorescein dyes with special reference to their use for the diagnosis of central nervous system tumors. Ann. Surg. 130:637-42

7. Hellan M., Spinoglio G., Pigazzi A. et. al. The influence of fluorescence imaging on the location of bowel transaction during robotic left sided colorectal surgery. Surg Endosc 2014; 28(5):1695-702

8. Lakowicz J.R., Szmacinski H., Novaczyc $\mathrm{K}$. et al. Fluorescence lifetime imaging of free and protein bound NADH. Prod Natl Acad Sci USA 1992; 89:1271-5

9. Jafari M.D., Lee K.H., Halabi W.J., Mills S.D., Carmichael J.C., Stamos M.J. et al. (2013) The use of indocyanine green fluorescence to assess anastomotic perfusion during robotic assisted laparoscopic rectal surgery. Surg Endosc 27(8):3003-3008

10. Miwa M. The principle of ICG fluorescence mode. Open Surg Oncol J 2010; 2:26-28

11. Zarrinpar A., Dutson E., Mobley C. et al. Intraoperative Laparoscopic Near Infrared Fluorescence Cholangiography to Facilitate Anatomical Identification: When to Give Indocyanine Green and How Much. Surgical Innovation 2016; 23(4);360-365

12. Boogerd L., Handgraaf H., Huurman A.L. et al. The Best Approach for Laparoscopic Fluorescence Cholangiography: Overview of the Literature and Optimization of Dose and dosing Time. Surgical innovation 2017; (ahead of print)

13. Souza A.V., Lin H., Henderson. E.R. et al. Review of fluorescence guided surgery systems: Identification of key performance capabilities beyond indocyanine green imaging. J. Biomed. Opt 2016; 21(8): 080901

14. Protyniak B. et al. Intraoperative indocyanine green fluorescence angiography - an objective evaluation of anastomotic perfusion in colorectal surgery. Am Surg. 2015; 81(6):580-4

15. Sherwinter D.A. Transanal near-infrared imaging of colorectal anastomotic perfusion. Surg Laparosc Endosc Percutan Tech .2012; 22:433-436

16. Cahill R.A., Anderson M., Wang L.M., Lindsey I., Cunningham C., Mortensen N.J. Near-infrared (NIR) laparoscopy for intraoperative lymphatic road-mapping and sentinel node identification during definitive surgical resection of early-stage colorectal neoplasia. Surg Endosc 2012; 26:197-204

17. Ryu S., Yoshida M., Ohdaira H. et al. A case of incarcerated femoral hernia with intestinal blood flow assessment by brightfield full-color near-infrared fluorescence camera: Report of a case. Int J Surg Case Rep. 2016; 29:234-236

18. Takahashi N., Nimura H., Fujita T. et al. Quantitative assessment of visual estimation of the infrared indocyanine green imaging of lymphnodes retrieved at sentinel node navigation surgery for gastric cancer. Medicine (Baltimore). 2016 Jun; 95(26):e4063.

19. Haslik W., Pluschnig U., Steger G.G. et al. Indocyanine green video angiography predicts outcome of extravasation injuries. PLoS One. 2014; 21;9(8):e103649

20. Sekijima M., Tojimbara T., Sato S. et al. 2004. An intraoperative fluorescent imaging system in organ transplantation. Transplant. Proc. 36:2188-90

21. Holm C., Mayr M.H., Hofter E. et al. 2002. Intraoperative evaluation of skin-flap viability using laser-induced fluorescence of indocyanine green. Br. J. Plastic Surg. 55:635-44

22. Detter C., Russ D., Iffland A. et al. 2002. Near-infrared fluorescence coronary angiography: a new noninvasive technology for intraoperative graft patency control. Proc. Heart Surg. Forum 5:364-69

23. Ferroli P., Nakaji P., Acerbi F. et al. 2011.
Indocyanine green (ICG) temporary clipping test to assess collateral circulation before venous sacrifice. World Neurosurg. 75:122-25

24. Yamaguchi S., De Lorenzi F., Petit J.Y. et al. 2004. The "perfusion map" of the unipedicled TRAM flap to reduce postoperative partial necrosis. Ann. Plastic Surg. 53:205-9

25. Azuma R., Morimoto Y., Masumoto K. et al. 2008. Detection of skin perforators by indocyanine green fluorescence nearly infrared angiography. Plastic Reconstr. Surg. 122:1062-67

26. Liu D.Z., Mathes D.W., Zenn M.R., Neligan P.C. 2011. The application of indocyanine green fluorescence angiography in plastic surgery. J. Reconstr. Microsurg. 27:355-64

27. Schaafsma B.E., Mieog J.S.D., Hutteman M. et al. The clinical use of indocyanine green as a nearinfrared fluorescent contrast agent for image-guided oncologic surgery. J. Surg. Oncol 2011. 104:323-32

28. Vettoretto N., Saronni C., Harbi A. et al. Critical view of Safety During Laparoscopic Cholecystectomy. JSJS 2011;15:322-325

29. Halbert C., Pagkratis S., Yang J. et al. Beyond the learning curve: Incidence of bile duct injuries following laparoscopic cholecystectomy normalize to open in the modern era. Surg. Endosc. 2016; 30(6):2239-43.

30. Billingsley K.G., Jarnagin W.R., Fong Y. et al. Segment-oriented hepatic resection in the management of malignant neoplasms of the liver. J.Am. Coll. Surg. 1998; 187:471481.

31. Harada N., Ishizawa T., Muraoka A. et al. Fluorescence navigation hepatectomy by visualization of localized cholestasis from bile duct tumor infiltration, J Am Coll Surg 2010;210:e2-6.

32. Aoki T., Murakami M., Yasuda D. et al. Intraoperative fluorescent imaging using indocyanine green for liver mapping and cholangiography, J. Hepatobiliary Pancreat Sci. 2010; 17:590-594

33. Kitajima T., Fujimoto Y., Hatano E. et al. Intraoperative fluorescent cholangiography using indocyanine green for laparoscopic fenestration of nonparasitic huge liver cysts. Asian J Endosc Surg. 2015; 8(1):71-4

34. Kahramangil B., Berber $E$. The use of near infrared fluorescence imaging in endocrine surgical procedures. J Surg Oncol.2017; 9999:1-8 